\title{
Left ventricular pseudo-aneurysm complicating a ruptured isolated congenital diverticulum
}

\author{
Liang Fang ${ }^{1}$, Chao Xue ${ }^{2}$, Ying Zhao ${ }^{2}$, Zhaoying $\mathrm{Wen}^{2}$, and Michael Henein ${ }^{3}$ \\ ${ }^{1}$ Chengde Medical College Affiliated Hospital \\ ${ }^{2}$ Capital Medical University Affiliated Anzhen Hospital \\ ${ }^{3}$ Umea University
}

April 28, 2020

\begin{abstract}
We report a case of 41-year-old woman who presented with chest tightness and shortness of breath. Transthoracic echocardiogram (TTE) showed left ventricular (LV) pseudo-aneurysm of the inferior wall with preserved LV systolic function. Coronary angiogram was normal. Surgical repair of the pseudo-aneurysm with a pericardial patch was performed, and pathological results confirmed rupture of an isolated congenital LV diverticulum.as the most likely etiology.
\end{abstract}

\section{Introduction}

Congenital left ventricular (LV) diverticulum is a rare cardiac anomaly which can be complicated with infective endocarditis, embolisms, arrhythmia and, rarely, rupture ${ }^{1-4}$. If ruptured, the outcome is usually poor with high mortality. We report a case of LV pseudo-aneurysm with ruptured diverticulum and successful surgical repaired.

A 41-year-old woman presented with a history of chest tightness and shortness of breath for 6 months. On clinical examination, blood pressure was 124/86 mmHg. The ECG showed sinus rhythm without evidence for ischemic changes, and chest X-ray revealed an enlarged heart. A transthoracic echocardiography (TTE) showed $20 \mathrm{~mm}$ discontinuity (with narrow connection neck) of basal segment of the inferior wall with 88 $\mathrm{mm} \times 71 \mathrm{~mm}$ echo-free space, suggesting pseudo-aneurysm formation wrapped within the pericardial (Fig 1A). There was a marked thrombus in the pseudo-aneurysm with large pericardial effusion (Fig 1B). Color Doppler demonstrated blood flow across the narrow neck of the pseudo-aneurysm (Fig 1C). Overall LV systolic function was preserved with no other combined abnormality.

The patient did not have any history of myocardial infarction, prolonged fever, chest trauma or any cardiac surgery. Coronary angiogram confirmed normal coronary arteries (Fig 2A \& 2B), and cardiac magnetic resonance imaging (MRI) confirmed the presence of the LV pseudo-aneurysm, on late gadolinium enhancement (LGE) showing LV pseudo-aneurysm with thin wall corresponding to fibrosis and/or scar (Fig 3A \& 3B).

The patient underwent surgery, during which a large pseudo-aneurysm of LV inferior wall was confirmed with a thrombus inside. The pseudo-aneurysm was closely adherent to the pericardium of the diaphragmatic surface (Fig 4). Surgical repair with a pericardial patch was performed, and postoperative TTE showed normal LV function with the patch located at the inferior wall. Pathological investigations showed the aneurysm size was $70 \times 60 \times 35 \mathrm{~mm}$. Based on the imaging, pathological results and medical history we considered that the ventricular aneurysm was a congenital LV diverticulum, which ruptured and caused a ventricular pseudo-aneurysm.

\section{Discussion}


Congenital LV diverticulum is a rare cardiac anomaly with $0.42 \%$ prevalence in adults and may range from 5 to $90 \mathrm{~mm}$, with normal LV systolic function ${ }^{5-7}$. It is thought to occur early in embryogenesis, emerging from an outer pouching of the endomyocardium through a weak LV segment. On cardiac imaging, LV diverticulum appears like an outer sac arising from the LV wall, the ratio of the connecting neck compared with the maximum diameter of the diverticulum varies, always less than $1^{7}$. LV diverticulum may be associated with midline thoracoabdominal defects, other congenital cardiac malformations, or can be isolated without intracardiac and extracardiac anomalies ${ }^{8,9}$. In isolated LV diverticulum, the patient is usually asymptomatic or may have overlapping symptoms of heart failure, chest pain, or dyspnea as seen in both aneurysms and pseudoaneurysms. And complications like embolism, infective endocarditis, arrhythmia and, rarely, rupture can be the initial presentation ${ }^{2,3,5}$.

Cardiac imaging is the most powerful tool for diagnosing LV diverticulum and for differentiating it from aneurysms especially pseudo-aneurysms, and delayed enhancement cardiac MRI is the most recently recommended ${ }^{10-12}$. LV diverticulum is usually classified into two types, fibrous and muscular types. Muscular type contains all three muscle layers with normal contractile function and is not prone to rupture, and is easier to differentiate from true-aneurysms which always accompany akinetic or dyskinetic wall complicating myocardial infarction. The fibrous type diverticulum, with complete absence of myocardium is usually located at the cardiac base or at the subvalvular area, making it difficult to differentiate from pseudo-aneurysms ${ }^{2,7,13}$. The LV pseudo-aneurysm is usually caused by cardiac rupture with discontinuity of the ventricular wall, and is encircled by adherent pericardium or scar tissue, with no myocardial tissue. The cause of pseudo-aneurysms includes acute myocardial infarction (AMI), endocarditis, trauma, iatrogenic operation (cardiac surgery, intervention), purulent pericarditis ${ }^{14-17}$. Over $50 \%$ of cases are due to AMI, and the most common location is the posterior and inferior wall.

Our patient denied any previous cardiac medical history, the coronary arteries were normal without any evidence for atherosclerotic disease and all other imaging investigations including; echocardiography, MRI, left ventriculography and surgery confirmed the presence of the LV pseudo-aneurysm. There were only few cardiomyocytes in the whole layer of the ventricular wall on histology. Based on the history and imaging findings, we believe that the most accurate diagnosis of this case is the ruptured LV diverticulum causing pseudo-aneurysm which was complicated with a large thrombus and pericardial effusion. Since the LV systolic function was preserved and the patient was hemodynamically stable, the surgical repair with patch was successful.

\section{References:}

1. Gowitt GT, Zaki SA. Rupture of a cardiac diverticulum resulting in sudden death. Am J Forensic Med Pathol. 1988;9(2):155-158.

2. Ohlow MA, von Korn H, Lauer B. Characteristics and outcome of congenital left ventricular aneurysm and diverticulum: Analysis of 809 cases published since 1816. Int J Cardiol. 2015;185:34-45.

3. Aquaro GD, Strata E, Di Bella G, et al. Prognostic role of isolated left ventricular diverticuli detected by cardiovascular magnetic resonance. J Cardiovasc Med (Hagerstown). 2015;16(8):562-567.

4. Westaby S, Katsumata T, Runciman M, Burch M. Ruptured left ventricular diverticulum in infancy. Ann Thorac Surg.1997;64(4):1181-1182.

5. Ohlow MA, Secknus MA, Geller JC, von Korn H, Lauer B. Prevalence and outcome of congenital left ventricular aneurysms and diverticula in an adult population. Cardiology. 2009;112(4):287-293.

6. Paz Y, Fridman E, Shakalia FM, Danieli J, Mishaly D. Repair of an isolated huge congenital left ventricular diverticulum. J Thorac Cardiovasc Surg. 2004;128(2):313-314.

7. Ohlow MA. Congenital left ventricular aneurysms and diverticula: definition, pathophysiology, clinical relevance and treatment.Cardiology. 2006;106(2):63-72. 
8. Ojha V, Chandrashekhara SH, Ganga KP, Saxena A, Gulati G. Congenital Left Ventricular Diverticulum in Pentalogy of Cantrell: Puzzle Solved With Dual-Source CT. Ann Thorac Surg. 2019;108(3):e205.

9. Li Q, Qu H, Wang H, Wang D, Li P, Liu T. Ventricular diverticulum: a review of the literature. J Card Surg. 2013;28(2):133-138.

10. Sharma A, Kumar S. Overview of left ventricular outpouchings on cardiac magnetic resonance imaging. Cardiovasc Diagn Ther.2015;5(6):464-470.

11. Srichai MB, Hecht EM, Kim DC, Jacobs JE. Ventricular diverticula on cardiac CT: more common than previously thought. AJR Am J Roentgenol. 2007;189(1):204-208.

12. Cianciulli TF, Del Carmen Gonzalez Colaso P, Saccheri MC, et al. Left ventricular diverticulum, a rare echocardiographic finding: two adult patients and review of the literature. Cardiol J.2009;16(1):76-81.

13. Marijon E, Ou P, Fermont L, et al. Diagnosis and outcome in congenital ventricular diverticulum and aneurysm. J Thorac Cardiovasc Surg. 2006;131(2):433-437.

14. Elshaer F, Altwijri F, Alrasheed F, Kharabsheh S, Habeeb W. Congenital ventricular diverticulum and MI - Diagnostic challenges and implications. J Saudi Heart Assoc. 2015;27(4):277-282.

15. Meng X, Yang YK, Yang KQ, et al. Clinical characteristics and outcomes of left ventricular pseudoaneurysm: A retrospective study in a single-center of China. Medicine (Baltimore). 2017;96(18):e6793.

16. Sunkara B, Briasoulis A, Afonso L, Ramappa P. Left ventricular pseudoaneurysm as a fatal complication of purulent pericarditis. Heart Lung. 2015;44(5):448-450.

17. Frances C, Romero A, Grady D. Left ventricular pseudoaneurysm.J Am Coll Cardiol. 1998;32(3):557-561.
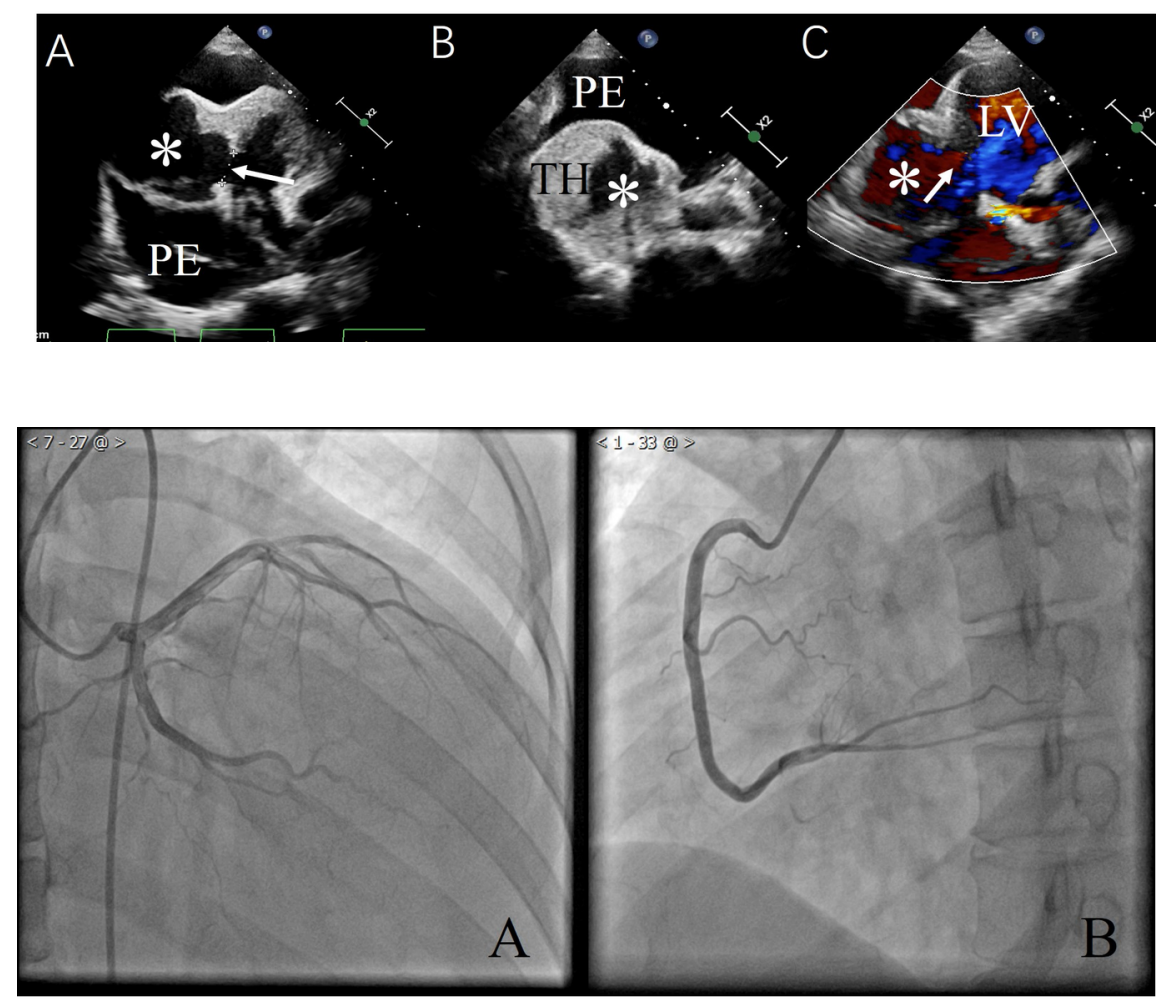

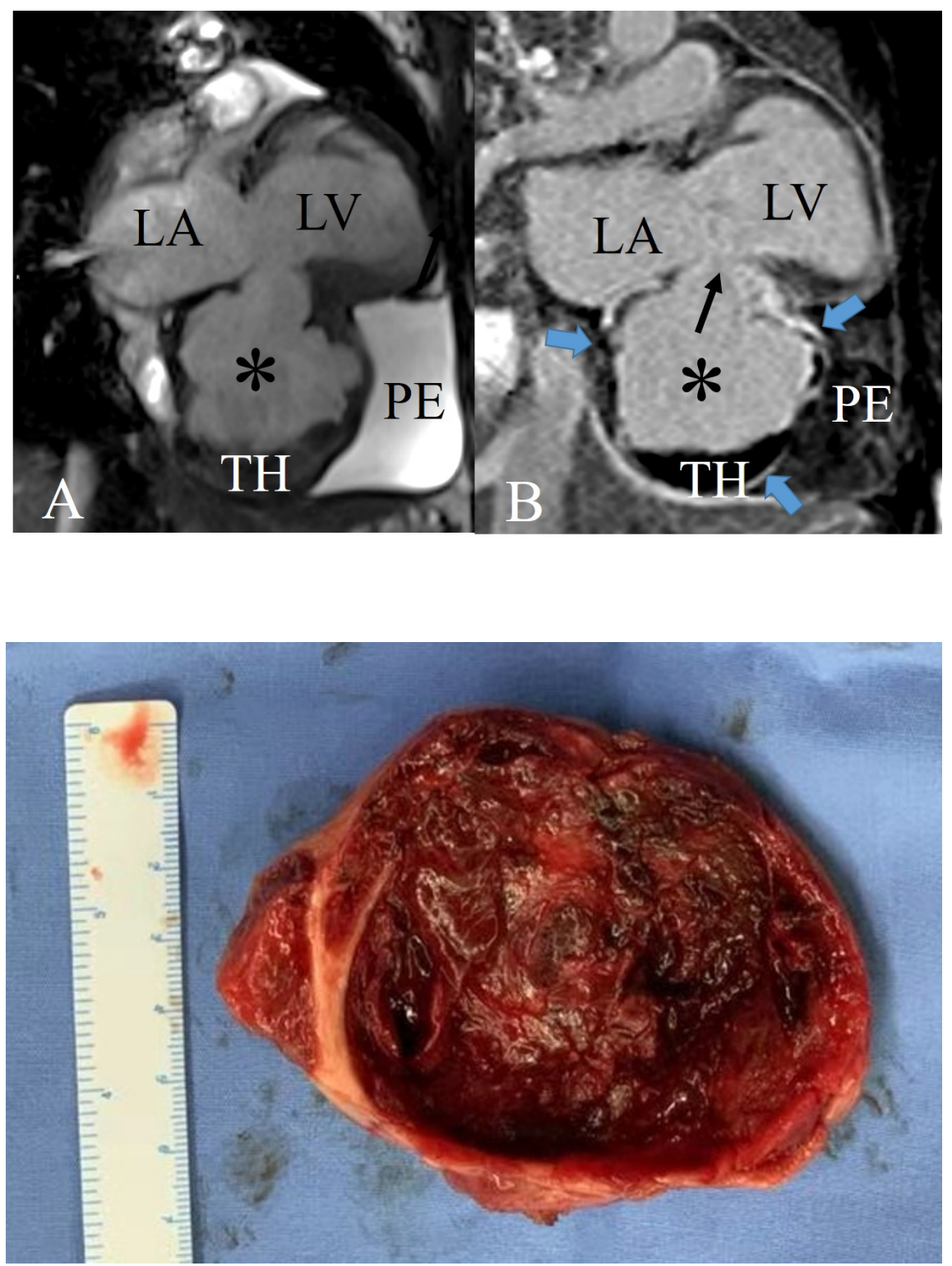